\title{
ATLAS End Cap Toroid Integration and Test
}

\author{
D. Elwyn Baynham, F. Steve Carr, Edward Holtom, Jarl Buskop, Alexey Dudarev, Philippe Benoit, \\ Giovanna Vandoni, Roger Ruber, Ruggero Pengo, and Herman H. J. Ten Kate
}

\begin{abstract}
The ATLAS Experiment at LHC, CERN will utilize a large, superconducting, air-cored toroidal magnet system with a long Barrel Toroid and two End Cap Toroids. Each End Cap Toroid will contain eight racetrack coils mounted as a single cold mass in a cryostat vessel of approximately $10 \mathrm{~m}$ diameter and $5 \mathrm{~m}$ width. The toroids provide the magnetic field for the muon detectors. The operating current is $20.5 \mathrm{kA}$ at $0.25 \mathrm{GJ}$ stored energy and a peak field of $4.1 \mathrm{~T}$ in the windings. This paper presents the status of the End Cap Toroid Project. Cold mass assembly and the integration of the full cold mass, 120 tons, into the vacuum cryostat for the first toroid are described. The specialized techniques, procedures and tooling infrastructure required for these operations are explained. The pre-installation cooldown to $77 \mathrm{~K}$ at the ATLAS cryogenic test facility is reported and the toroid installation in the ATLAS Experiment 100m underground in the ATLAS cavern will be reviewed.
\end{abstract}

Index Terms-Detector magnet, superconducting, toroidal field.

\section{INTRODUCTION}

$\mathbf{T}$ HE ATLAS muon spectrometer is based on a superconducting toroid magnet system [1] consisting of a long Barrel Toroid (BT) and two End Cap Toroids (ECT's). The two End Cap Toroids are designated ECTA and ECTC according to their position in the ATLAS experiment. The major features of the End Cap Toroid Magnets have been described elsewhere [2]. Each ECT consists of a cold mass, eight superconducting coil modules and eight keystone boxes, mounted in a large castellated cryostat [3]. The cold mass will be directly cooled by pumped circulation of two phase helium. All services will be carried through a single turret. In this paper we describe the assembly of the two cold masses at CERN and the integration of the first full cold mass into the vacuum cryostat to form the final toroid assembly of ECTA. The planning and infrastructure for pre-installation cooldown to $77 \mathrm{~K}$ at the ATLAS test facility is described.

\section{Cold Mass ASSEMBly}

The Cold Mass consists of eight superconducting coils and eight keystone box (KSB) modules. The keystone box modules

Manuscript received August 25, 2006. This work was funded by the ATLAS Collaboration, NIKHEF and CERN.

D. E. Baynham, F. S. Carr, and E. Holtom are with the CCLRC Rutherford Appleton Laboratory, Chilton, Oxon OX11 0QX, UK (e-mail: e.baynham@rl.ac.uk).

J. Buskop is with NIKHEF, 1009 DB Amsterdam, the Netherlands.

A. Dudarev, P. Benoit, G. Vandoni, R. Ruber, R. Pengo, and H. H. J. Ten Kate are with CERN, CH-1211, Geneva 23, Switzerland.

Color versions of one or more of the figures in this paper are available online at http://ieeexplore.ieee.org.

Digital Object Identifier 10.1109/TASC.2007.897734

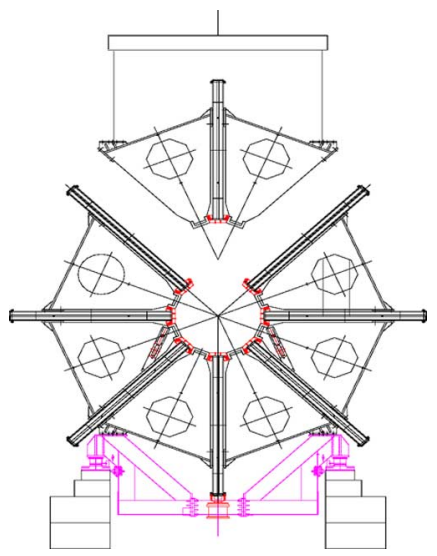

Fig. 1. Cold mass assembly concept at CERN.

are the structural elements that maintain the toroidal shape under gravitational and magnetic loads. The cold mass components were manufactured by BRUSH HMA BV in the Netherlands under a contract placed by NIKHEF, Amsterdam. Coil and keystone box fabrication has been described previously [2].

Cold mass assembly at CERN has been made with the toroid axis horizontal, see Fig. 1. Cold mass assembly was undertaken in three main phases.

\section{A. Preparatory Work on Coils and Keystone Boxes}

Extensive preparatory work was carried out on both coils and keystone boxes. A key part of this work was the preparation, installation and testing of cooling circuits. A dual cooling circuit system is installed to minimize the impact of any leak in cooling circuit component.

Aluminum cooling circuits were installed in the coils at the fabrication stage and bonded into the structure during coil impregnation. After delivery to CERN aluminum to stainless steel transitions were welded on all coil circuits in order to make final connections to the stainless steel distribution manifold. Cooling circuits were fabricated and installed in the keystone boxes. Circuits were subjected to rigorous $\mathrm{x}$-ray inspection, thermal cycling and leak testing before release to the cold mass assembly process. Coil units were prepared for installation of quench heaters, thermal sensors, superconducting quench detectors (SQDs) and voltage taps for quench detection and joint monitoring.

When the coils and keystone boxes are assembled to form a complete cold mass it is important to locate the coils accurately at the 45 degree azimuthal spacing and correct radial position in order to achieve accurate toroidal field profiles. It is also important to ensure a correct mechanical connection interface between the coils and keystone boxes, firstly, to transfer gravitational loads and secondly, to transfer the magnetic loads. 


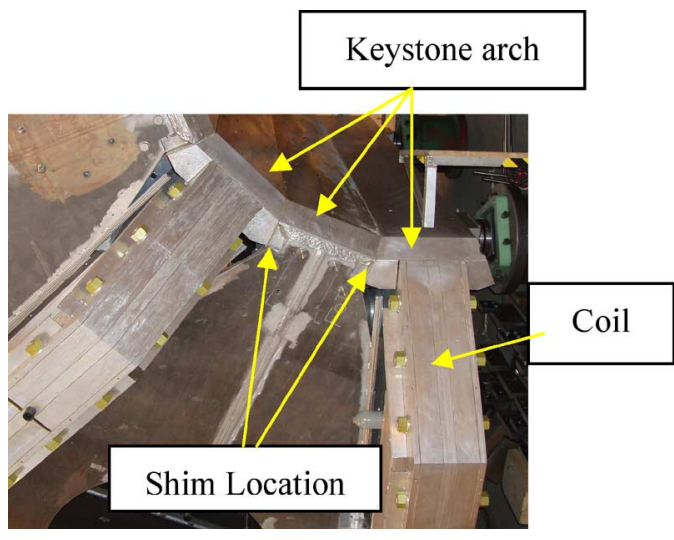

Fig. 2. Keystone arch force transfer concept showing shim location.

Fig. 2 shows the load transfer concept at the inner circumference. At the design stage spaces were left between the coils and the keystone boxes, $5 \mathrm{~mm}$ at the inner radius and $10 \mathrm{~mm}$ at the outer radius. These spaces were designed to allow accurate geometric assembly taking account of the manufacturing tolerances. In order to ensure the mechanical integrity of the assembly and allow uniform force transfer the interfaces were shimmed in preparation for the final assembly stage.

The shim process was carried out in two stages. The coils and keystone boxes were first aligned in a frame with the toroid axis vertical. In this process the coils were placed at accurate 45 degree azimuthal and radial positions using survey bushes. With the coils set and the keystone box installed shim spaces were measured at localized positions on the main flange interfaces. This was repeated for all module positions.

The second stage was to prepare the shims over the extended flange faces. This was achieved by forming a sandwich of thixotropic epoxy (a gel epoxy which flows freely under pressure) and aluminum plates and deforming this to the correct interface thickness previously measured before curing the epoxy.

With this preparation procedure the coils and keystone boxes could be accurately re-assembled with axis horizontal to the defined geometry. Final bonding with a thin resin layer ensured high quality force transfer surfaces. This work has been completed for both cold masses.

\section{B. Cold Mass Geometric Assembly}

A sequential build of the cold mass for ECTA was made on the toroid integration rails. As an initial step the first coil (lower) was assembled with its two mating keystone boxes.

Further sub-assemblies of coils and keystone boxes were made in preparation for final assembly. These sub-assemblies were added sequentially, located by special blocks and finally glued and bolted at the flange interfaces. This process continued until the mid plane coils (horizontal) were in place. A dry assembly of the top half cold mass sub-assemblies was then made. This dry assembly allowed in-situ confirmation of the fit-up of the final parts in the keystone arch. During this build up coil positions were checked by survey.The top half of the cold mass pre-assembly was then stripped back and the vacuum vessel bore tube and thermal shield installed in the

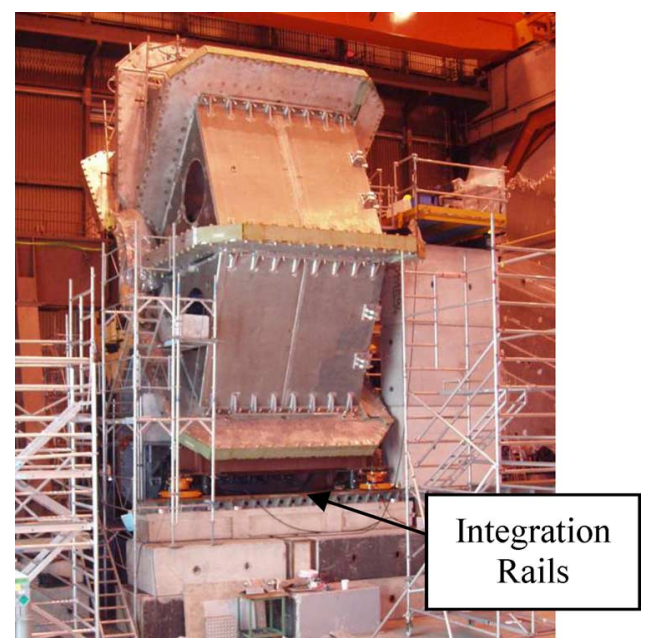

Fig. 3. Full assembly of cold mass on integration rails.

cold mass bore. The final step in the assembly process was the re-positioning, final gluing and bolting of all elements in the top half of the cold mass assembly. At this stage final blocking and bolting at all flange interfaces was made. Fig. 3 shows the final mechanical assembly of ECTA cold mass mounted on the rail infrastructure in the integration hall.The preparations for final assembly of ECTC cold mass have proceeded in parallel in an adjacent building. With the knowledge gained from the first assembly the procedure for the second cold mass was modified to allow maximum pre-assembly and minimum final assembly work on the integration rails at high level. This process has been based on the full pre-assembly of the top and bottom halves of ECTC cold mass. These will be transported to the integration rails as complete units. The half-assemblies already have the main cooling circuit manifolds installed and the conductor joints between coils fabricated. This will really shorten the final assembly steps on the integration rails.

\section{Instrumentation, Electrical Connection, Cooling Circuit Manifolding}

As the build of cold mass ECTA progressed the electrical connections were made between coils. A conductor tail from each coil was formed across the end of the keystone box and the conductors welded to form the low resistance connection. The joints were clamped and bonded to the KSB structure to give mechanical and thermal stability.

During the cold mass build the cooling circuits, 4 per coil, 4 per keystone box were connected through a stainless steel manifold system inside the keystone box structure. The manifolds were pre-formed and assembled into sub-elements for final installation to ensure a minimum number of in-situ welds in the keystone box structure. All circuits were successfully pressure and leak tested.

The cold mass has been fully equipped with instrumentation for temperature monitoring, quench detection, joint resistance monitoring and quench initiation. Quench initiation will be made by $2 \times 100 \mathrm{~W}$ heaters on each coil. These heaters were bonded directly to the aluminum stabilized conductor at the feed/exit from each coil. 


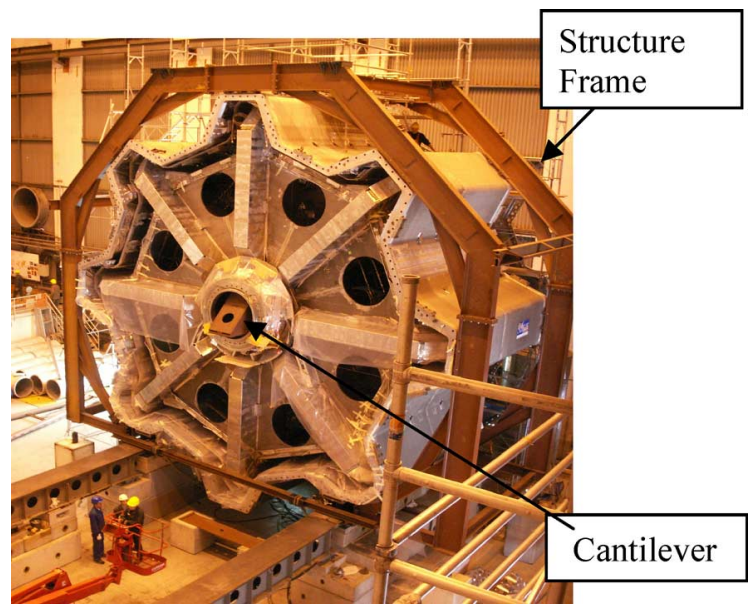

Fig. 4. Integration of vacuum vessel shell over cold mass. Cold mass supported on cantilever beam. Vessel shell supported by large structure frame on integration rails.

\section{TOROID INTEGRATION}

Toroid Integration comprises 4 main steps; transfer of the complete cold mass onto the large cantilever beam and loading from the gravity support rods; movement of the outer vacuum vessel shell over the cold mass to allow connection of axial force transfer rods and thermal shield end plates; installation of final instrumentation and the services turret; closure of the vessel end plates and transfer of the overall load to the rails. Transfer of the 130 ton cold mass into the $10 \mathrm{~m}$ diameter vacuum vessel requires a set of heavy tooling which was pre-installed in B191 at CERN. The tooling comprises a large cantilever beam and a pair of assembly rails which allow the large components to be moved on air pads. This section describes the main integration steps and the status.

\section{A. Transfer Onto the Cantilever}

The cold mass was assembled on the mid point of the rails on a special support frame. When the cold mass was complete the frame with the cold mass was moved over the large cantilever beam using air pads. At this stage the gravity support rods were installed to connect the cold mass and center bore tube. The gravity support rods are fabricated in stainless steel with a main section $60 \mathrm{~mm}$ by $125 \mathrm{~mm}$. In operation each rod will carry a load of approximately 40 tons. The rods are connected to a special saddle on the vacuum bore tube and a bracket on the keystone box through rotating ball ends. With the bore tube supported on the cantilever the full cold mass weight was transferred to the cantilever and the support frame removed. During this process the deflection of the cantilever was compensated by jacks to maintain the axis of the cryostat bore tube horizontal. The whole process required very careful alignment of the cold mass to ensure equal loading of the gravity support rods. Through this adjustment a load sharing of better than $10 \%$ was achieved. Fig. 4 shows the full cold mass of ECTA mounted on the cantilever.

\section{B. Integration With Outer Vessel Shell and AFT Rods}

Before transferring the cold mass onto the cantilever frame one vacuum vessel end plate was transferred to the inner part of the cantilever frame to be available for final vessel closure.

The outer vessel shell was then transferred over the cold mass using the rail and air pad system. During this operation the basic shape of the outer shell was maintained by a large external frame, see Fig. 4 which shows the outer shell positioned over the cold mass.

In operation the cold mass of each ECT will experience an axial force of approximately 280 tons towards the center of the experiment due to the interaction with the Barrel Toroid (BT) field. Within the ECT cryostat this force is transferred to the vacuum vessel shell by 16 axial force tie (AFT) rods. Each rod is connected to the cold mass keystone box and to a special bracket on the inside of the vacuum vessel shell. On the outer surface of the vacuum vessel shell a bracket will be installed to react this load to the BT coil cryostats. The shape of the vessel shell and its alignment with the cold mass axis and the vessel bore tube was critical for the correct setting of the AFT rod lengths. Distortions of the vessel after set up could lead to large stresses in the AFT rod system and by linkage give unequal loading in the gravity support rod system. It was found that the outer shell shape was difficult to control to a tolerance better than $20 \mathrm{~mm}$ without the end plates of the vessel closed and fixed by the dowel system. Such errors were sufficient to give problems with final AFT rod set up. After extensive analysis and manipulation of the vessel shell it was decided to implement a system to allow adjustment of the AFT rods after closure of the vessel end plates and which even allows adjustment after cooldown. In such a way assembly errors, mounting errors and any differential cooldown errors can be eliminated. This modified system is under implementation.

\section{Installation of Thermal Shields and Services Turret}

The thermal shield and superinsulation components were preinstalled in the vacuum vessel shell as a first stage of integration. The completion of the thermal shield system involves the installation of stay tube shields, the shield end plates and the connection of all elements, see Fig. 5. The cooling of the thermal shields is provided by dual circuits attached to each end plate. The bore tube, stay tube and outer shell shields are cooled by conduction from the end plates. The shield end plates are fabricated as two half plates which will be finally closed by coil cover plates.

The half endplates have been installed using specially designed tooling to handle the components which measure approximately $10 \mathrm{~m}$ by $5 \mathrm{~m}$, see Fig. 5 . Installation of the stay tube shields and the thermal and mechanical connections to the end plate cooling is in progress.

All services to the ECT's are connected through a single turret mounted on the top vacuum vessel castellation. The turret structure consists of an outer shell, a large top plate and a sealing ring to complete the vacuum integrity. The system is designed to allow installation of the top plate with all components before closing with the outer shell and the sealing ring. Current connections are made through two $20 \mathrm{kA}$ current leads mounted through the top plate on a bellows system to accommodate the cooldown shrinkage of the cold mass. Cryogenic connections 


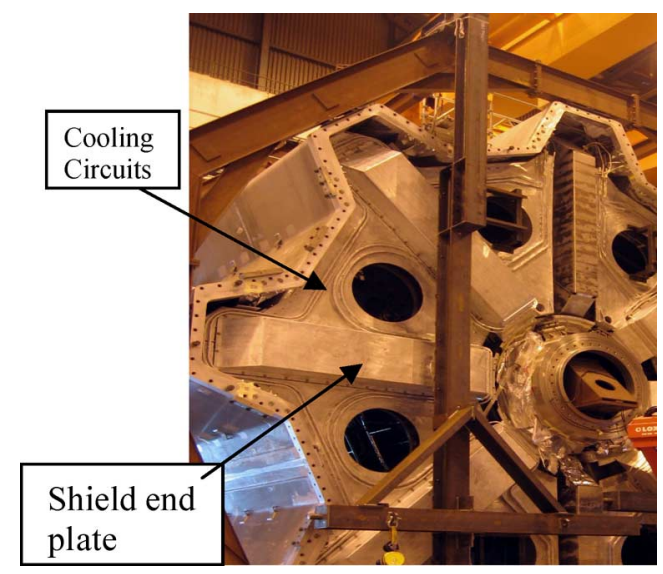

Fig. 5. Installation of end plate thermal shields.

are made through a single vacuum barrier system which will incorporate liquid helium feed and return circuits at $4.5 \mathrm{~K}$ in addition to helium gas feed and return circuits cooling the thermal shield system at $40 \mathrm{~K}$ and $80 \mathrm{~K}$ respectively. The ports which will carry the instrumentation interface plates are also mounted on the top plate. Two diffusion pumps will be mounted on opposing sides of the turret shell.

A full pre-build of the top plate with all components fitted was made at ground level to minimize the amount of assembly work to be undertaken at the top of the vacuum vessel. The top plate, with all components, has been lifted into position and work is in progress to make the final connections to the coil conductor tails and the various cooling circuits. At this stage the final connections to instrumentation in the vessel are being made. When complete the turret vessel will be closed by fitting the outer shell equipped with the vacuum pumps.

\section{Final Integration Closure of Vessel}

This is a complex task which requires installation of the vessel stay tubes, the interfacing to the bore tube of the vessel and the outer shell. Final mechanical closure involves the installation of 96 large dowels in each end plate. The dowels will transfer the vacuum loads (750 tons) from the outer shell to the endplate and locate the two parts to better than $0.1 \mathrm{~mm}$. When the end plates are fitted the vacuum integrity will be checked by pumping between the double o-ring system.

When the vessel is complete the full toroid will be transferred from the cantilever to the rail system using jacks and air-pads. The toroid can then be prepared for transport to the test station.

\section{PReparation for Test AND InStallation}

A cooldown of each toroid to $77 \mathrm{~K}$ will be made before installation. The test will be carried out at the ATLAS cryogenic test facility previously used to test and commission the ATLAS Barrel Toroid coils. This test will confirm the correct operation of vacuum systems, cooling circuits and instrumentation. Full leak checking and voltage testing will be carried out. The infrastructure for cooldown and test is close to completion.

When the toroid is installed in the ATLAS cavern the services; cryogens, vacuum, power and instrumentation, will be connected to the top level of the ATLAS experiment through a services tower approximately $5 \mathrm{~m}$ in height. The tower design is complete and the flexible chain to connect to the fixed services at the side of the ATLAS cavern is under procurement. The chain system will allow the services to remain connected to the End Cap Toroid when the magnet is moved from the beam position to allow access to the inner part of the ATLAS experiment.

\section{SCHEDULE}

Integration of the first Toroid ECTA is scheduled for completion in September 2006. A pre-cooling test to $77 \mathrm{~K}$ will be performed before transfer to the ATLAS cavern in late 2006.

The second toroid, ECTC, will follow with approximately six months interval.

\section{ACKNOWLEDGMENT}

The authors acknowledge the contributions of many colleagues and co-workers at CERN and the Rutherford Appleton Laboratory.

\section{REFERENCES}

[1] H. H. J. Ten Kate, "The ATLAS superconducting magnet system: Status of construction and installation," IEEE Trans. Appl. Superconductivity, vol. 16, p. 499, 2006.

[2] D. E. Baynham et al., "ATLAS End Cap TOROID magnets cold mass design and manufacturing status," IEEE Trans. Appl. Superconductivity, vol. 14, p. 485, 2004.

[3] D. E. Baynham et al., "ATLAS End Cap Toroid cold mass and cryostat integration," IEEE Trans. Appl. Superconductivity, vol. 16, p. 537, 2006. 